GP152 MANAGEMENT OF MULTIDOSE LIQUID ORAL FORMS IN FRENCH PAEDIATRICS HOSPITALS

AGAR Julien*, Sophie Brafine, Anne Fratta, Yannick Ho. Hôpital A Trousseau, Paris, France

10.1136/archdischild-2019-epa.216

Multidose liquid oral forms (MLOFs) are the most appropriate for paediatric population. They allow a better adaptability of the doses and facilitate the administration to patients who cannot swallow tablets or capsules. Many questions come up concerning the management in hospital pharmacies, the use in care services or the fate of the bottles after patient discharge.

The aim of this study was to conduct a state of play of MLOFs management in French paediatrics hospitals.

An online 3-part questionnaire was sent to 20 French paediatrics hospital pharmacies. The first part focuses on the management in the pharmacy (nominative or global dispensing). The second part concerns practices in care services (bottle sharing between patients, fate after patient discharge), and the last part is about existing procedures and documents to frame the practices. The collection period ran from June to October 2018.

17 among the 20 reached hospital pharmacies answered the questionnaire (85\%). 16/17 of this pharmacies dispense MLOFs both nominative and global (drug staffing) ways, 1 establishment practices nominative dispensation only. When the dispensation is nominative, most of the pharmacies dispense the entire bottle of the drug: $14 / 17$ when there is a marketing authorization (MA), 15/17 when the drug is under 'Autorisation Temporaire d'Utilisation' (ATU) a French disposition when there is no existing form with MA in France. 2 institutions produce oral syringes within the pharmacy. In care services, 12/17 hospitals allow vials sharing between patients, and only 4 out of 12 have a limited authorized list of services, pharmaceutical specialities or situations (isolation...). Regarding to the administration devices used, 4/12 use the provided device, 11/12 enteral syringes, 1/12 oral syringes and $1 / 12$ parenteral syringes. When the patient is discharged, MLOFs with MA are most often discarded (11/17) unlike ATU wich are often given to the patient (15/17). The management policy is validated by the nursing care division in 9 out of 17 institutions. On the other hand, few centers have proper use documents as its disposal: 6/12 for sharing vials, $7 / 17$ for equivalence tables and $7 / 17$ conducts audits on MLOFs use.

This work highlight a real heterogeneity in the MLOFs management in French paediatrics hospitals. The issues are shared between pharmacists, and it would seem useful to share available documents and knowledge. The next step of this work would be to propose harmonized procedures that could be adopted by most of the paediatrics institutions.

\section{GP153 DEMAND ANALYSIS OF REGIONAL PAEDIATRIC HIGH DEPENDENCY UNIT (PHDU- CRITICAL CARE LEVEL II): LESSONS FROM IRISH EXPERIENCE FOR A DECADE}

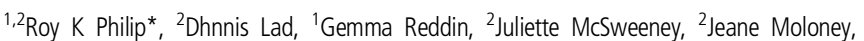
${ }^{1}$ Maaz Mirza, ${ }^{2}$ Catherine Nix, ${ }^{2}$ Trina Dooley, ${ }^{1}$ Ailish Hannigan. ${ }^{1}$ Graduate Entry Medical School, University of |Limerick, Limerick, Ireland; '2University Hospital Limerick, Limerick, Ireland
}

10.1136/archdischild-2019-epa.217
Background Paediatric High Dependency Units (PHDU) specialize in the safe and optimal care of children requiring more close observation and intervention than can be provided on the general ward.

Aims 1. To analyze the patient characteristics, seasonal trends, bed occupancy rate and length of stay (LOS) of regional PHDU. 2. To develop a demand model for PHDU based on an annual regional birth rate and a childhood population ( $<16$ years) for the Mid-West of Ireland and to propose National and European projections.

Methods Bed occupancy analysis of PHDU (critical care level II) at University Hospital Limerick (UHL) was conducted. Daily assessment records covering over nine years (2010 to 2019) was used in which patient information indicating age, gender, symptom/signs at presentation along with the management received in PHDU were obtained. Additionally, a recorded location for admission/discharge, date and time of admission and discharge and transfer to tertiary centres was documented. Data was verified with computerized hospital inpatient enquiry (HIPE) and analyzed using SPSS. Demand modelling based on birth cohort and childhood population of the region was determined. UL Hospital group research and ethics committee approved the study.

Results Over our study period annual mean general admission rate of 117 to PHDU was observed. Respiratory and Neurological presentations were the most common morbidities $(32 \%$ and $27 \%$ respectively) followed by endocrine, sepsis related and surgical. $53 \%$ of admissions were male and $46 \%$ female with commonest age group was 0-4 years. Median length of stay was 2.3 days. Commonest respiratory morbidity was bronchiolitis and the most frequent acute neurological presentation was prolonged convulsion/status Annual mean rate for additional respiratory support (mostly Nasal CPAP/BiPAP/High Flow Humidified Oxygen) was 28 and that of tertiary care critical care transfers 18 . Since the opening of PHDU in Limerick, tertiary care transfers to Dublin decreased by $54 \%$ and admissions to adult ICU by $78 \%$. We estimated a mean annual paediatric admission demand of 110 per 100,000 childhood population $<16$ years and 9 infants/1,000 live births annually. Conclusion Our review provides strong evidence for rationalization in the development of more PHDU (critical care level II) facilities available at the regional centres. Additionally, our observations highlight the PHDU demand, appropriate equipping and multidisciplinary staff training/competence to safely cater for the critically ill infants and children at regional centres, thus supporting their safe care closer to home with obvious health economic and societal benefits.

\section{GP154 IMPROVING ASTHMA SEVERITY AND CONTROL SCREENING IN A LARGE URBAN HEALTH SYSTEM}

Marina Reznik*, Philip Ozuah. Montefiore Medical Center, Bronx, USA

10.1136/archdischild-2019-epa.218

Background Asthma severity and level of control classification is the first step in asthma management. To optimize asthma management, screening must be done routinely and in a standardized manner at the time of patient's visit with the health care provider.

Objectives 1) To develop and implement asthma severity and control screening within electronic health record (EHR) system of a large urban health system to improve pediatric asthma 
care; 2) To increase asthma screening from $0 \%$ to $80 \%$ by December 2020.

Methods We developed an algorithm for asthma screening in EHR based on national guidelines that asked about medications taken at home, exercise-induced symptoms, medication adherence, daytime and night-time symptoms, use of rescue medication and interference with activity in the past 4 weeks, and use of oral corticosteroids in the past year. Screening was conducted by the nursing staff prior to patient being seen by the provider. Based on responses to the screening questions, severity/control classification is calculated and displayed in EHR for provider to then treat patient accordingly. Classic Quality Improvement tools such as the Model for Improvement and Plan Do Study Act (PDSA) cycles were used to incorporate changes into daily workflow. Practice Advisory Board met monthly to discuss barriers to implementation and identify solutions. The Board made decisions on adopting successful strategies and brainstormed new strategies to address barriers.

Results This project is ongoing and preliminary results are available from 3 practices: small pediatric, large pediatric and family medicine practices. The ultimate goal is to improve care and clinical outcomes of children with asthma. To date, a total of 4,057 screenings have been completed at these sites. Screening rates improved from $0 \%$ to $78.6 \%$ in a small pediatric practice, from $0 \%$ to $66.7 \%$ in a large pediatric practice, and from $0 \%$ to $38.7 \%$ in a family practice. Several problems were encountered during the process of change: competition with other nurse-completed screenings; screening questions are available in English only within EHR; nursing staff shortages; nurses forgetting to conduct screening; and caregiver not knowing information about child's asthma or disagreeing with asthma diagnosis.

Conclusion We developed an asthma screening algorithm within EHR, trained nursing staff and implemented patient screening at the time of clinic visit allowing providers take action on asthma management based on the screening result. Further collaborative efforts are needed to improve and sustain screening rates with the ultimate goal of improving patient clinical outcomes.

\section{GP155 ARE CLINICAL PREDICTION RULES IN PAEDIATRICS VALIDATED? A LITERATURE REVIEW}

CA Keegan Hickey*, ZA Sarani, MB O'Neill. Mayo University Hospital, Castlebar, Ireland

10.1136/archdischild-2019-epa.219

Aims To identify and appraise the geographical and temporal transportability of externally validated clinical prediction rules (CPRs) in the paediatric population.

Methods A Medline search was conducted from 2000-2015 for publications involving the derivation, internal or external validation of a CPR in children aged $0-21$ years. Validation studies were included if they described a CPR which was developed within this time frame.

Results 193 studies were identified for inclusion. The selected studies describe 133 CPRs, across more than 10 paediatric specialities. 63 CPRs were not validated (47\%). Of the 70 validated rules identified (53\%), 25 were internally validated, 8 were cross validated and 53 were externally validated. Only 13 CPRs (10\%) were both internally and externally validated and 21 CPRs (19\%) were externally validated more than once. 15 internally validated studies used split sample methodology while only 5 performed bootstrapping regression methods. Of the 87 studies measuring external validity, the majority of studies (66\%) were performed at a different site to the derivation. Only 5 studies were performed at the same site while 22 did not disclose this information. 43 externally validated studies included populations that were recruited in a different country to the derivation and 27 of those were on a different continent. Only two externally validated studies were shown not to have temporal transportability. Performance of the rules in validation was poorly reported, and if reported usually underperformed in comparison to the original.

Conclusion While many CPRs are developed, they are often not validated externally. Of those that have been validated, it is often difficult to uphold the same level of performance. The majority of externally validated CPRs assessed in this study meet geographical and temporal transportability which is a crucial feature in the CPR's ability to uphold performance amongst different populations.

\section{GP156 INCREASING RATES OF SELF-HARM AMONG CHILDREN 10-14 IN IRELAND: A TEN-YEAR NATIONAL REGISTRY STUDY 2007-2016}

\footnotetext{
${ }^{1,2,3}$ Fiona McNicholas* ${ }^{*}{ }^{4,5}$ Helen Keeley, ${ }^{5,6}$ eve griffin, ${ }^{6}$ Elaine McMahon, ${ }^{6,7}$ Paul Corcoran, 7,6Ivan J Perry, 7,6Ella Arensman. ${ }^{1}$ UCD, Dublin, Ireland; ${ }^{2}$ OLCHC, Dublin, Ireland; ${ }^{3}$ Lucena Clinic SJOG, Dublin, Ireland; ${ }^{4}$ CAMHS HSE South Cork, Cork, Ireland; ${ }^{5}$ UCC, Cork, Ireland; ${ }^{6}$ National Suicide Research Foundation, Cork, Ireland; ' 5 School Public Health UCC, Cork, Ireland
}

\subsection{6/archdischild-2019-epa.220}

Despite stabilization in suicide rates in most countries, rates of Self Harm have continued to increase in young people. Research abounds as to the risks and protective factors but there is still a huge gap in our knowledge as to what leads one person to act on suicidal ideation and another to refrain, and what causes increasing numbers to engage in repetitive non-suicidal self-(NSSI).

The current study examined trends in rates and trends of self-harm among young people in Ireland aged 10-14 years over a ten-year period.

Methods Data from the National Self-Harm Registry Ireland on presentations to hospital emergency departments (EDs) following self-harm in 10-14 years-old during the period 2007-2016 were included. We calculated annual self-harm rates per 100,000 by age, sex and method of self-harm. Poisson Regression models were used to examine trends in rates.

Results The rate of self-harm was 72 per 100,000 (34 for males and 112 for females) an increase of $75 \%$ between 2007 and 2016 (IRR $=1.75,95 \% \mathrm{CI}=1.15-2.10)$, higher in boys $(82 \%$ versus $72 \%)$. Rates of attempted hanging and selfcutting more than doubled ( $I R R=2.43$ and 2.72 , respectively). Attendances to hospital with self-harm increased over the course of the day peaking at $11 \mathrm{pm}$, and and unexpectedly perhaps highest on Mondays.

Presentations involving self-poisoning were highest, and Paracetamol containing medication were involved in the majority of presentations (51\%), 19\% involved NSAIDs and $12 \%$ involved minor tranquilisers (22\% for boys and 9\%) for girls). $8 \%$ of presentations involved alcohol. Considering those discharged from the ED, the majority $(57 \%)$ were referred to 